\title{
Ig Kappa Chain V-I Region D-13
}

National Cancer Institute

\section{Source}

National Cancer Institute. Ig Kappa Chain V-I Region D-13. NCI Thesaurus. Code

C117125.

Ig kappa chain V-I region D-13 is encoded by the human IGKV1D-13 gene. This protein is involved in the mediation of antibody-antigen specificity. 